Original Article

\title{
Predictive Parameters of Febrile Neutropenia and Clinical Significance of G-CSF Receptor Signaling Pathway in the Development of Neutropenia during R-CHOP Chemotherapy with Prophylactic Pegfilgrastim in Patients with Diffuse Large B-Cell Lymphoma
}

\author{
Do Young Kim ${ }^{(D 1}{ }^{1}$, Jehyun Nam ${ }^{(D 2}{ }^{2}$, Joo-Seop Chung ${ }^{1}$, Byeol Eun Jeon ${ }^{2}$, Ji Hyun Lee ${ }^{3}$, Jae-Cheol Jo ${ }^{4}$, Sang-Woo Kim (iD) ${ }^{2}$, Ho-Jin Shin ${ }^{(D)}$ \\ ${ }^{1}$ Division of Hematology-Oncology, Department of Internal Medicine, Biochemical Research Institution, Pusan National University Hospital, \\ Pusan National University School of Medicine, Busan, ${ }^{2}$ Department of Biological Sciences, Pusan National University, Busan, \\ ${ }^{3}$ Department of Internal Medicine, Dong-A University College of Medicine, Busan, ${ }^{4}$ Division of Hematology-Oncology, Department of \\ Internal Medicine, Ulsan University Hospital, Ulsan, Korea
}

Purpose Pegfilgrastim is widely used to prevent chemotherapy-induced neutropenia (CIN) and febrile neutropenia (FN) in patients with diffuse large B-cell lymphoma (DLBCL). We investigated the predictive factors affecting CIN and FN incidence in patients with DLBCL receiving rituximab, cyclophosphamide, doxorubicin, vincristine, and prednisone (R-CHOP) chemotherapy with pegfilgrastim and conducted experiments to find reason for the occurrence of CIN even when pegfilgrastim was used.

Materials and Methods We reviewed the CIN and FN events of 200 patients with DLBCL. Based on these data, we investigate the association with predictive factor and the levels of granulocyte-colony stimulating factor (G-CSF) receptor signaling pathway markers (pSTAT3, pAKT, pERK1/2, pBAD, and CXCR4) in bone marrow (BM) samples isolated from patients with DLBCL.

Results FN was significantly associated with stage III/IV (hazard ratio [HR], 12.74) and low serum albumin levels (HR, 3.87). Additionally, patients with FN had lower progression-free survival (PFS; 2-year PFS, $51.1 \%$ vs. 74.0\%) and overall survival (OS; 2-year OS, $58.2 \%$ vs. $85.0 \%$ ) compared to those without FN. The occurrence of CIN was associated with overexpression of G-CSF receptor signaling pathway markers, and expression levels of these markers were upregulated in BM cells co-cultured with DLBCL cells. The rate of neutrophil apoptosis was also higher in neutrophils co-cultured with DLBCL cells and was further promoted by treatment with doxorubicin.

Conclusion Our findings suggest that high DLBCL burden may alter the BM environment and G-CSF receptor signaling pathway, even in chemotherapy-naïve state, which may increase CIN frequency during R-CHOP chemotherapy.

Key words Neutropenia, G-CSF receptor, Chemotherapy, Pegfilgrastim, Diffuse large B-cell lymphoma

\section{Introduction}

Diffuse large B-cell lymphoma (DLBCL) is the most common histological subtype of non-Hodgkin lymphoma (NHL) [1]. Although the treatment outcomes of DLBCL have drastically improved since the introduction of rituximab, an antiCD20 monoclonal antibody, in the early 2000s [2,3], chemotherapy-induced neutropenia (CIN) and febrile neutropenia (FN) continue to be responsible for treatment-related deaths $[4,5]$. In addition, the success of curative treatment can be compromised by reduced or delayed chemotherapy after CIN $[6,7]$.

To prevent these adverse events, current guidelines recom- mend the prophylactic use of recombinant human granulocyte-colony stimulating factor (G-CSF) [8,9], which reduces the incidence of CIN $[10,11]$ and acts primarily by activating the G-CSF receptor. Although the specific mechanisms underlying G-CSF signaling remain unclear, some intracellular signaling pathways such as Janus kinase (JAK)/signal transducer and activator of transcription protein (STAT) and phosphatidylinositol 3-kinase (PI3K)/protein kinase B (AKT) have been shown to be involved in apoptosis and cell survival and differentiation $[12,13]$.

Pegfilgrastim is a long-acting form of G-CSF that is synthesized by adding a polyethylene glycol molecule to G-CSF (filgrastim) to reduce the proteolytic degradation and renal
Correspondence: Sang-Woo Kim
Department of Biological Sciences, Pusan National University,
2 Busandaehak-ro 63beon-gil, Geumjeong-gu, Busan 46241, Korea
Tel: 82-51-510-2260 Fax: 82-51-581-2962 E-mail: kimsw@pusan.ac.kr

\author{
Co-correspondence: Ho-Jin Shin \\ Division of Hematology-Oncology, Department of Internal Medicine, Biomedical \\ Research Institute, Pusan National University Hospital, Pusan National \\ University School of Medicine, 179 Gudeok-ro, Seo-gu, Busan 49241, Korea \\ Tel: 82-51-240-7839 Fax: 82-51-256-8330 E-mail: hojinja@hanmail.net \\ *Do Young Kim and Jehyun Nam contributed equally to this work.
}


clearance of filgrastim, resulting in a more stable molecule with a reduced need for frequent dosing. Furthermore, the neutrophil-regulated clearance mechanism maintains a constant concentration of pegfilgrastim in the body until it has recovered from neutropenia [14].

Studies have shown that pegfilgrastim can reduce neutropenia-related complications, including CIN and FN, when used as a primary prophylactic after chemotherapy. Additionally, treatment once-per-cycle with pegfilgrastim was found to yield more favorable results than a daily regimen of filgrastim administration $[15,16]$. Consequently, pegfilgrastim has been used widely to prevent CIN in cancer patients; however, there is limited data regarding the efficacy of pegfilgrastim to prevent $\mathrm{FN}$ in cancer patients receiving chemotherapy, particularly patients with DLBCL receiving rituximab, cyclophosphamide, doxorubicin, vincristine, and prednisone (R-CHOP) immunochemotherapy.

A previous study found that $48 \%$ of the patients with DLBCL experienced grade 3/4 neutropenia and 16\% developed neutropenic fever during R-CHOP chemotherapy with pegfilgrastim [17]. Therefore, we aimed to identify predictive factors affecting the incidence of CIN and FN during R-CHOP chemotherapy with pegfilgrastim in patients with DLBCL and to find out why prophylactic pegfilgrastim does not prevent CIN during chemotherapy. To achieve these goals, we investigated the molecular and cellular roles of G-CSF receptor signaling in CIN during chemotherapy with prophylactic pegfilgrastim treatment in patients with DLBCL.

\section{Materials and Methods}

\section{Patients and samples}

We reviewed the medical records of 200 patients with DLBCL who underwent R-CHOP chemotherapy followed by prophylactic pegfilgrastim treatment from three medical centers in South Korea. We collected comprehensive baseline patient characteristics, including disease- and host-related factors, as well as data about CIN and FN events after the first cycle of R-CHOP chemotherapy and treatment outcomes. Since prophylactic pegfilgrastim treatment has been covered by the National Health Insurance system in South Korea since 2015, all patients diagnosed with DLBCL between 2015 and 2018 were enrolled in the study. All enrolled patients received $\mathrm{R}-\mathrm{CHOP}$ chemotherapy at an initial dose determined according to their characteristics and disease status, with pegfilgrastim administered at least 24 hours after the end of chemotherapy. Blood samples for laboratory testing were collected on day 1 and again $7 \pm 3$ days after chemotherapy to determine the occurrence of neutropenia and the nadir value of absolute neutrophil count (ANC). Daily
Table 1. Study patient demographics and clinical characteristics Characteristic

No. of patients $(\%)$

\begin{tabular}{|c|c|}
\hline Age, median (range, yr) & $62(16-88)$ \\
\hline \multicolumn{2}{|l|}{ Sex } \\
\hline Male & $118(59.0)$ \\
\hline Female & $82(41.0)$ \\
\hline \multicolumn{2}{|l|}{ ECOG } \\
\hline $0-1$ & $165(82.5)$ \\
\hline$\geq 2$ & $34(17.0)$ \\
\hline Unknown & $1(0.5)$ \\
\hline \multicolumn{2}{|l|}{ IPI risk group } \\
\hline Low $(0-1)$ & $68(34.0)$ \\
\hline Low-intermediate (2) & $44(22.0)$ \\
\hline High-intermediate (3) & $43(21.5)$ \\
\hline High (4-5) & $44(22.0)$ \\
\hline Unknown & $1(0.5)$ \\
\hline \multicolumn{2}{|l|}{ Ann Arbor stage } \\
\hline I & $23(11.5)$ \\
\hline II & $55(27.5)$ \\
\hline III & $24(12.0)$ \\
\hline IV & $98(49.0)$ \\
\hline B symptoms & $52(26.0)$ \\
\hline Bulky disease & $26(13.0)$ \\
\hline Extranodal involvement & $126(63.0)$ \\
\hline Single lesion & $62(49.2)$ \\
\hline Lesion $\geq 2$ & $64(50.8)$ \\
\hline LDH elevation & $133(66.5)$ \\
\hline \multicolumn{2}{|l|}{ Hans criteria for DLBCL } \\
\hline GCB & $53(26.5)$ \\
\hline Non-GCB & $127(63.5)$ \\
\hline Unknown & $20(10.0)$ \\
\hline \multicolumn{2}{|l|}{ Double express } \\
\hline None & $7(3.5)$ \\
\hline Double & $31(15.5)$ \\
\hline Unknown & $162(81.0)$ \\
\hline Bone marrow involvement & $34(17.0)$ \\
\hline Median cellularity $(\%)$ & 40 \\
\hline \multicolumn{2}{|l|}{ Chromosome } \\
\hline Normal & $155(77.5)$ \\
\hline Abnormal & $34(17.0)$ \\
\hline Unknown & $11(5.5)$ \\
\hline \multicolumn{2}{|l|}{ Initial chemotherapy dose } \\
\hline Standard & $125(62.5)$ \\
\hline Reduced & $75(37.5)$ \\
\hline
\end{tabular}

DLBCL, diffuse large B-cell lymphoma; ECOG, Eastern Cooperative Oncology Group; GCB, germinal center B-cell; IPI, International Prognostic Index; LDH, lactate dehydrogenase.

laboratory tests were performed for inpatients, and for outpatients, laboratory tests were performed at outpatient clinic visits scheduled 1 week after chemotherapy. CIN is generally 

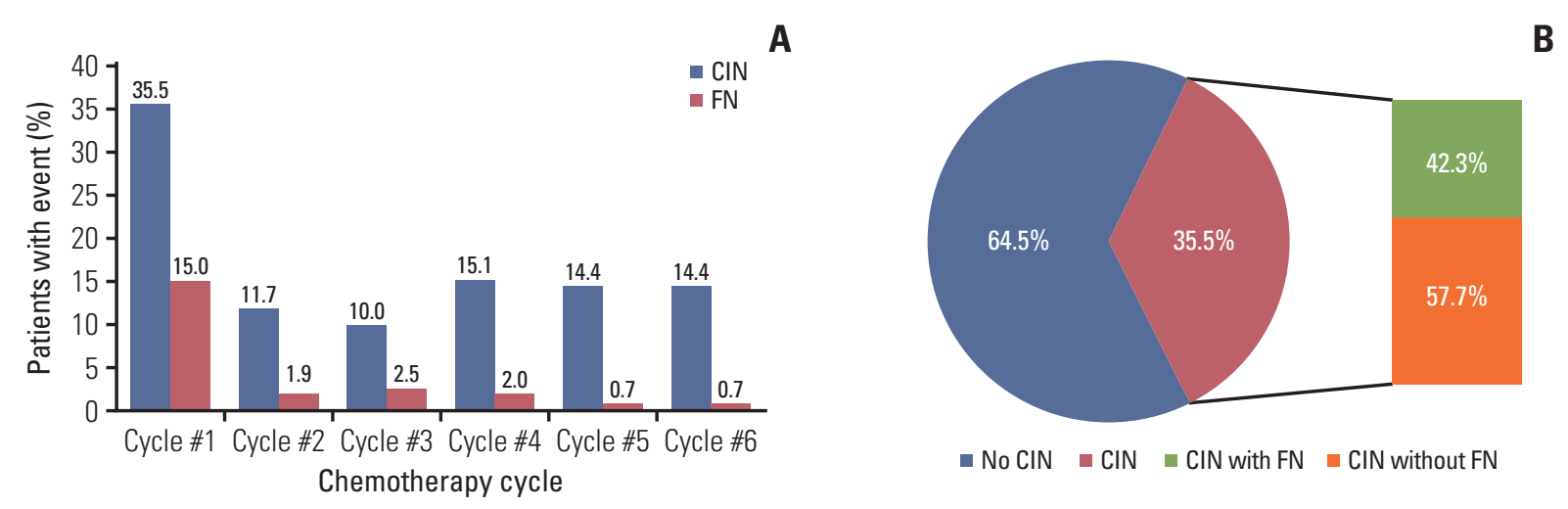

Fig. 1. CIN and FN events. (A) CIN and FN events on each R-CHOP chemotherapy cycle. (B) Analysis of CIN and FN events at first chemotherapy cycle of diffuse large B-cell lymphoma patients. CIN, chemotherapy-induced neutropenia; FN, febrile neutropenia; R-CHOP, rituximab, cyclophosphamide, doxorubicin, vincristine, and prednisone.

characterized as a decreased $\mathrm{ANC}<2,000 / \mu \mathrm{L}$ in peripheral blood. In this study, grade $3 / 4$ (ANC $<1,000 / \mu \mathrm{L}$ ) neutropenia was defined as CIN. Fever was defined as a single oral temperature measurement of $\geq 38.3^{\circ} \mathrm{C}$, or a temperature of $\geq 38^{\circ} \mathrm{C}$ sustained over a 1 -hour period. FN was defined as the occurrence of fever in a state of neutropenia with an ANC of $<500 / \mu \mathrm{L}$ or $<1,000 / \mu \mathrm{L}$ that was expected to decrease to $<$ $500 / \mu \mathrm{L}$ within 48 hours.

\section{Cell culture and antibodies}

Three different cell lines (human bone marrow [BM], murine BM, and OCI-Ly-1 [RRID: CVCL_1879]) were used in this study. Human BM samples from patients with DLBCL were aspirated prior to the first dose of R-CHOP chemotherapy, cryopreserved in the National Biobank of Korea, and analyzed after thawing according to the appropriate guidelines. Human bioresources deposited in the National Biobank of Korea must be accompanied by 'Consent to Research on Human Materials' or 'Consent to Donation on Human Materials' in accordance with 'Bioethics and Safety Act Enforcement Rules.'

Murine BM cells were collected from 7-week-old C57BL / 6 mice and red blood cells were removed using ammonium chloride solution. BM cells were then resuspended in Dulbecco's modified Eagle medium (cat No. \#LM001-05, Welgene, Gyeongsan, Korea), supplemented with $10 \%$ fetal bovine serum (FBS; cat No. \#SH30084.03, HyClone Laboratories, Logan, UT) and incubated overnight at $37^{\circ} \mathrm{C}$ in $5 \% \mathrm{CO}_{2}$.

The OCI-Ly1 cell line was kindly provided by Dr. Ricardo Aguiar from the University of Texas Health Science Center at San Antonio and maintained in Roswell Park Memorial Institute-1640 medium (cat No. \#30027.01, HyClone Laboratories) supplemented with $10 \% \mathrm{FBS}$ at $37^{\circ} \mathrm{C}$ in $5 \% \mathrm{CO}_{2}$. The OCI-Ly1 cell line was authenticated using short tandem repeat profiling (Cosmogen Tech., Seoul, Korea) within the last 3 years and all experiments were performed using mycoplasma-free cells.

The following antibodies were used in this study: antiphospho AKT (1:1,000 dilution, cat No. \#9271, Cell Signaling Technology [CST], Danvers, MA), anti-phospho STAT3 (1:1,000, cat No. \#9131S, CST), anti-phospho extracellular signal-regulated kinase (ERK) 1/2 (1:1,000, cat No. \#9101, CST), anti-phospho BCL2-associated agonist of cell death (BAD; 1:1,000, cat No. \#9295, CST), and anti-C-X-C chemokine receptor type 4 (CXCR4; 1:1,000, cat No. \#ab1670, Abcam, Burlingame, CA).

\section{Western blotting}

Protein levels were determined by western blotting. Briefly, cells were harvested, washed with $1 \times$ phosphate-buffered saline, and lysed in radioimmunoprecipitation assay buffer (Elpis Biotech, Daejeon, Korea) mixed with sodium vanadate (1 mM, Sigma-Aldrich, Merck KGaA, Darmstadt, Germany), $\beta$-glycerol phosphate (50 mM, Sigma-Aldrich, Merck KGaA), protease inhibitor (1×, G-Biosciences, St. Louis, MO), EDTA (5 mM, G-Biosciences), and $\beta$-mercaptoethanol (142 mM, Bioworld Technology, St. Louis Park, MN). Lysates were resolved on $10 \%$ or $15 \%$ polyacrylamide gels, transferred onto polyvinylidene difluoride membranes, and then blocked with $1 \%$ bovine serum albumin (MP Biomedicals, Irvine, CA) reconstituted in Tris-buffered saline containing $0.1 \%$ Tween 20 (TBST) for 1 hour at room temperature. The membranes were probed overnight with primary antibodies at $4^{\circ} \mathrm{C}$, followed by incubation with horseradish peroxidase-conjugated secondary antibodies for 1 hour at room temperature. Signals were then visualized using enhanced chemiluminescent reagent (EzWestLumi plus, ATTO, Tokyo, Japan) and protein levels were analyzed using Lumino- 


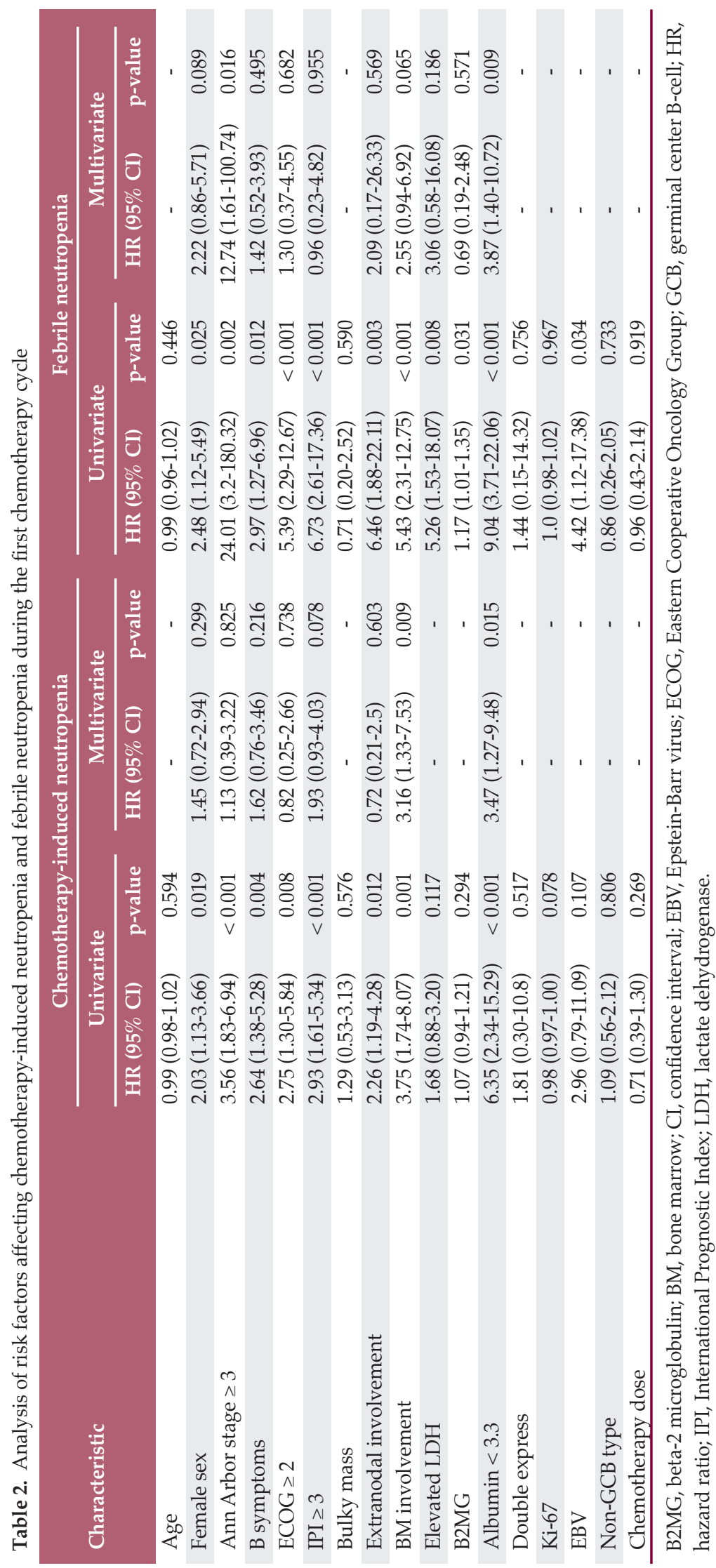




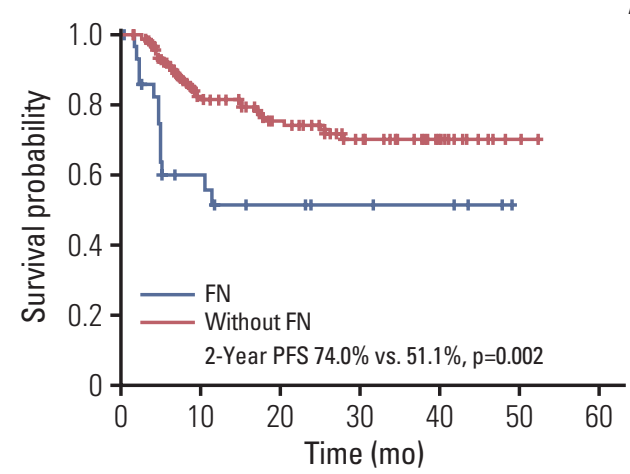

A

B
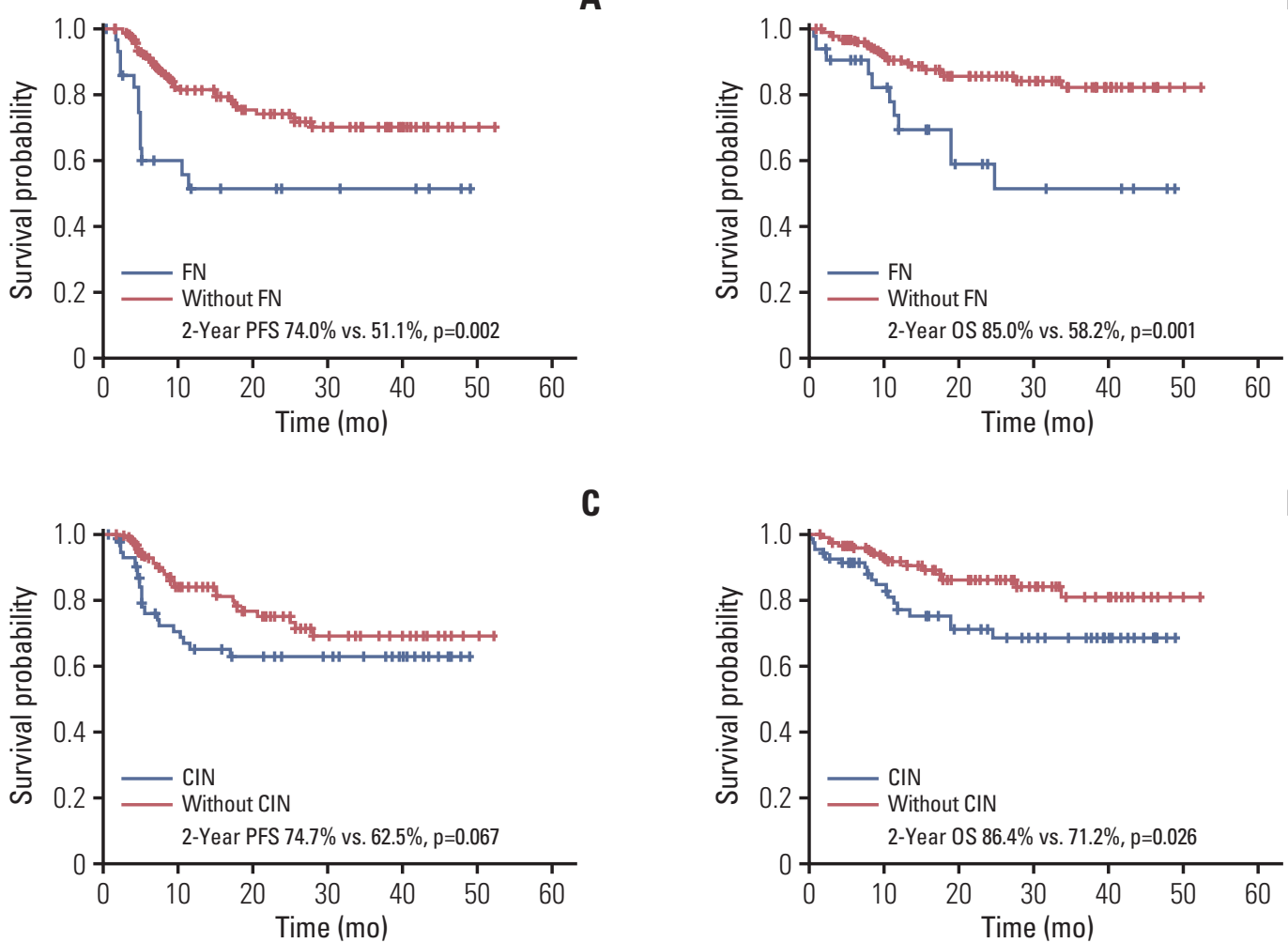

C

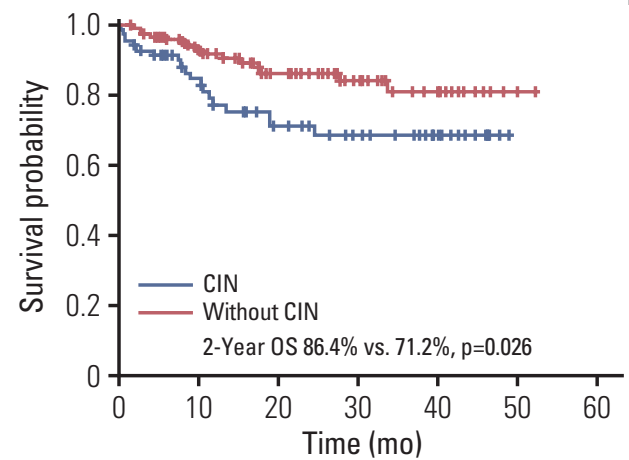

Fig. 2. Effect of CIN and FN on the progression-free survival (PFS, A and C) and overall survival (OS, B and D) Kaplan-Meier curves showing the PFS and OS of patients with newly diagnosed diffuse large B-cell lymphoma according to the FN events (A, B) and CIN (C, D) in the first cycle of chemotherapy. CIN, chemotherapy-induced neutropenia; FN, febrile neutropenia; OS, overall survival; PFS, progressionfree survival.

Graph II image analysis software (WSE-6100, ATTO). Protein expression was quantified relative to $\beta$-actin using ImageJ v1.52a. All western blotting data were the result of at least three independent experiments.

\section{Flow cytometry}

The rate of apoptosis was investigated in murine BM cells co-cultured with OCI-Ly1 cells and murine BM cells alone with or without chemotherapy. To determine whether DLBCL cells affected neutrophil apoptosis during chemotherapy, $1 \times 10^{7}$ cells $/ \mathrm{mL}$ murine BM cells were co-cultured with $5 \times 10^{6}$ OCI-Ly1 cells in 6-well Transwell plates and incubated at $37^{\circ} \mathrm{C}$ for 24 hours. The cells were then treated with 2.5 or $5.0 \mu \mathrm{M}$ of doxorubicin and incubated for a further 24 hours under the same conditions. Next, BM cells were harvested and stained with fluorescein isothiocyanate-conjugated rat anti-CD11b (cat No. \#561688, BD Biosciences, San Jose, CA), allophycocyanin-conjugated rat anti-mouse Ly-6G (cat No. \#560599, BD Biosciences), and propidium iodide (cat No. \#51-66211E, BD Biosciences) according to the manufacturer's instructions. Neutrophils were identified using key cell sur- face markers $\left(\mathrm{CD} 11 \mathrm{~b}^{+} \mathrm{Ly}_{6 \mathrm{G}}\right)$ and apoptosis was determined by propidium iodide using flow cytometry (BD FACSCanto II software).

\section{Statistical analysis}

Patient characteristics were described using descriptive statistics. Categorical variables were compared using the chisquare test or Fisher exact test, whereas continuous variables were examined using a t test or $\mathrm{u}$ test depending on the data distribution. Stepwise conditional logistic regression analysis was used to control for the effects of confounding variables and identify independent risk factors for CIN and FN. Meaningful risk factors with a p-value of $<0.05$ at the univariate level were included in the multivariate logistic model. Kaplan-Meier survival analysis was used to generate survival curves, which were compared using the log-rank test. All clinical data analyses were performed using SPSS ver. 22.0 (IBM Corp., Armonk, NY). All in vivo data were analyzed using one-way analysis of variance in Microsoft Excel (Microsoft, Redmond, WA) and Prism (GraphPad, San Diego, CA). p-values $<0.05$ were considered significant. 


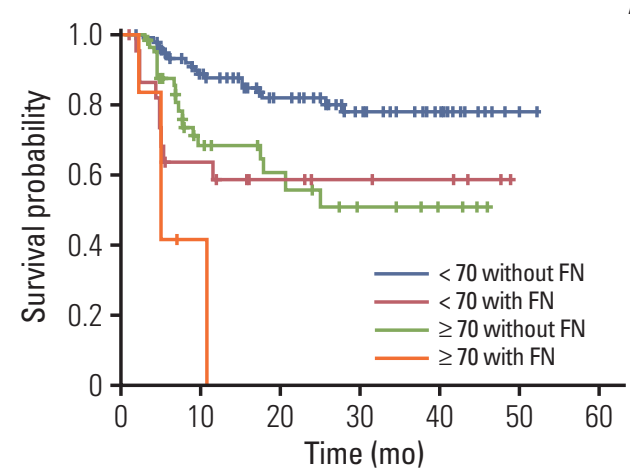

A
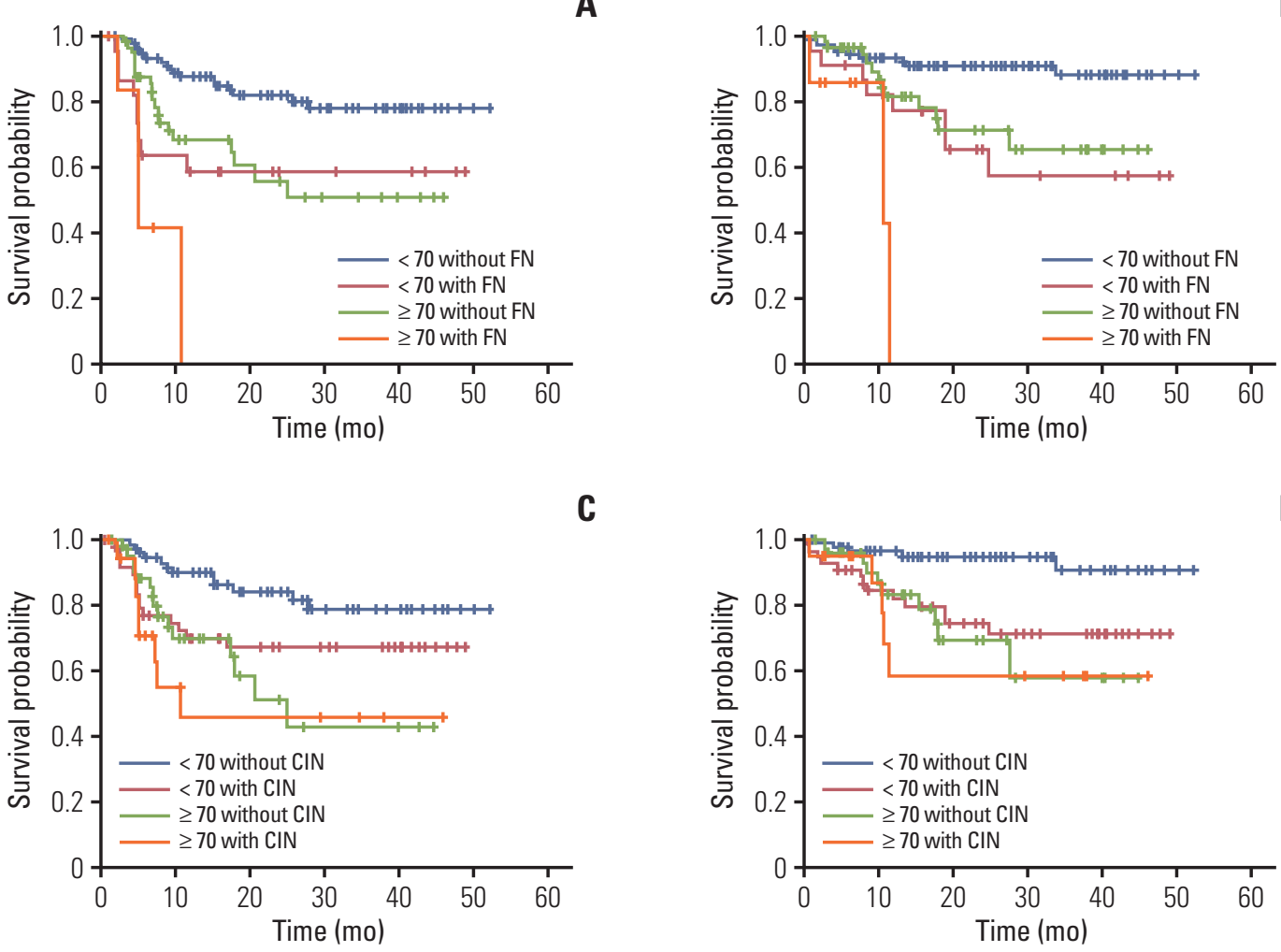

C

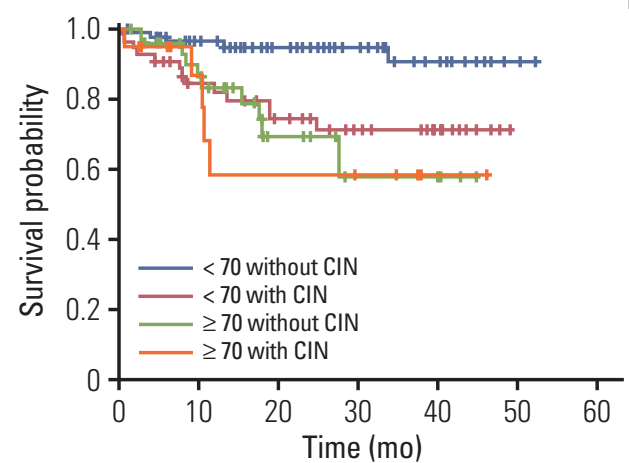

Fig. 3. Effect of CIN and FN on the progression-free survival (PFS, A and C) and overall survival (OS, B and D) according to patient's age. Kaplan-Meier curves showing the PFS and OS of patients with newly diagnosed diffuse large B-cell lymphoma according to the age and CIN (A, B) or FN events (C, D) in the first cycle of chemotherapy. CIN, chemotherapy-induced neutropenia; FN, febrile neutropenia; OS, overall survival; PFS, progression-free survival.

\section{Results}

\section{Patient characteristics}

The median age of the 200 DLBCL patients included in this study - at the time of diagnosis-was 62 years (range, 16 to 88 years) and 165 patients $(82.5 \%)$ had an Eastern Cooperative Oncology Group (ECOG) performance status of 0 or 1 . According to the International Prognostic Index (IPI), 87 (43.5\%) patients were in high-intermediate or highrisk groups and approximately half of the patients $(n=98$, 49\%) had Ann Arbor stage IV disease. Fifty-two patients (26.0\%) experienced B symptoms and $126(63.0 \%)$ manifested extranodal lesions. BM infiltration of lymphoma cells was confirmed in 34 patients $(17.0 \%)$ and the median BM cellularity was $40 \%$. Thirty-four patients $(17.0 \%)$ presented abnormal chromosome test results. According to Han's criteria, 53 patients $(26.5 \%)$ had germinal center B-cell-like (GCB) DLBCL and 127 (63.5\%) had non-GCB DLBCL. Owing to old age or poor general condition, 75 patients $(37.5 \%)$ received a reduced dose of chemotherapy (Table 1$)$.

\section{CIN and predictive factors}

Among the 200 patients enrolled in this study, initial CIN and FN events were commonly observed during the earlier treatment cycles, particularly after the first chemotherapy cycle. Seventy-one patients $(35.5 \%)$ experienced CIN during their first chemotherapy cycle, among which $30(15.0 \%)$ experienced FN; however, the incidence of CIN and FN was lower during subsequent chemotherapy cycles (Fig. 1).

Univariate analysis revealed that female sex, stage III/IV, B symptoms, poor performance status, IPI, extranodal involvement, BM involvement, and low albumin levels correlated significantly with CIN. Moreover, multivariate analysis indicated that BM involvement (hazard ratio [HR], 3.16; 95\% confidence interval [CI], 1.33 to $7.53 ; \mathrm{p}=0.009)$ and low albumin levels (HR, 3.47; 95\% CI, 1.27 to 9.48; $\mathrm{p}=0.015$ ) was associated with CIN events.

Further univariate analysis indicated that female sex, stage III/IV, B symptoms, ECOG performance status, IPI, extranodal involvement, BM involvement, elevated lactate dehydrogenase, $\beta 2$-microglobulin, and low serum albumin levels were strong predictive factors for $\mathrm{FN}$, among which stage 

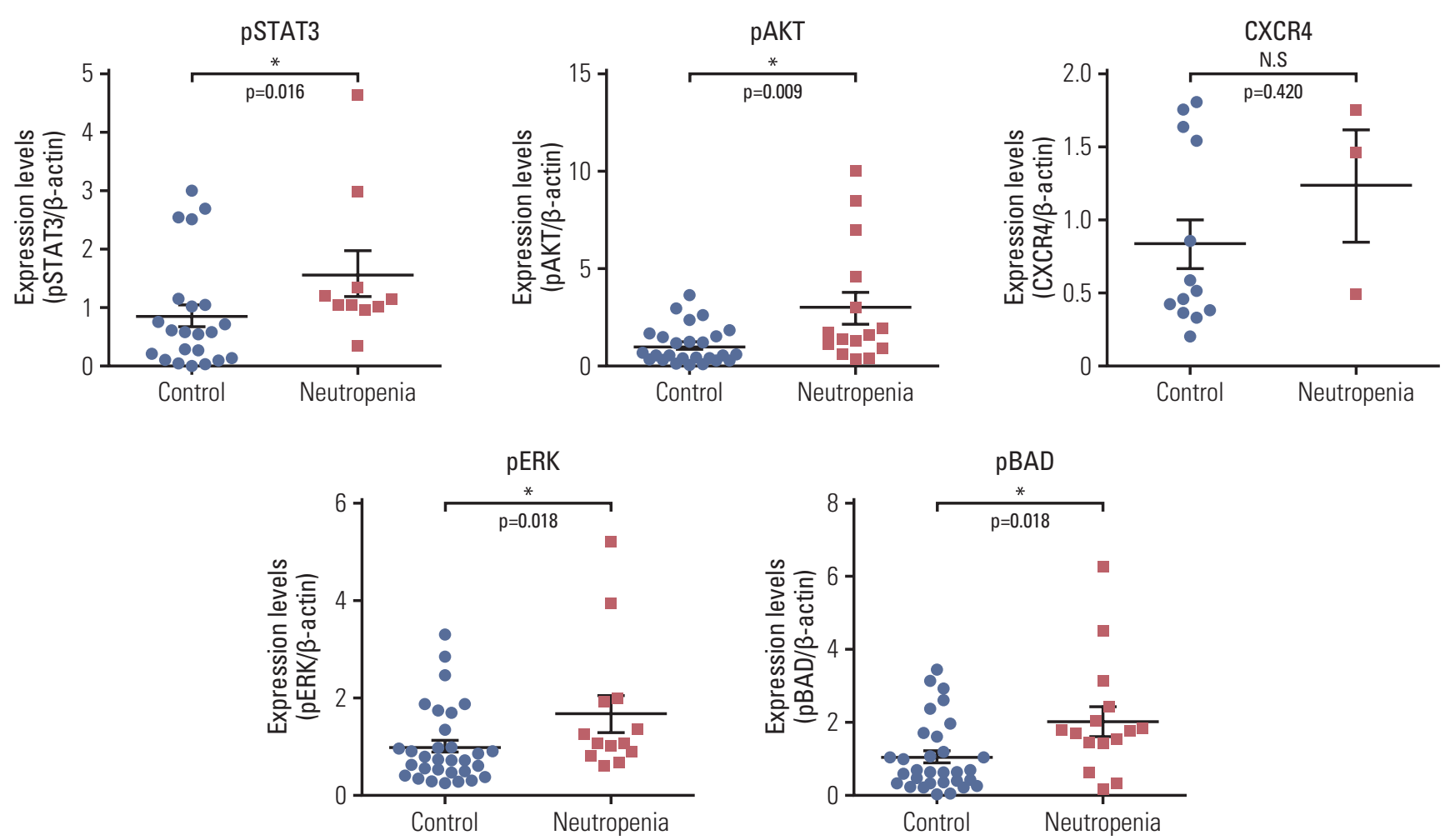

Fig. 4. Levels of phospho-STAT3, phospho-AKT, phospho-ERK, phospho-BAD, and CXCR4 in bone marrow samples from DLBCL patients with neutropenia were higher than in those without. Western blot analysis of phospho-STAT3, phospho-AKT, phospho-ERK1/2, phospho-BAD and CXCR4 levels in bone marrow samples of DLBCL patients with or without CIN were performed. Band densities of the western blots were quantified by Image software and densitometry results were shown as a dot graph. Each graph represents as mean \pm SD. AKT, protein kinase B; BAD, BCL2-associated agonist of cell death; CIN, chemotherapy-induced neutropenia; CXCR4, C-X-C chemokine receptor type 4; DLBCL, diffuse large B-cell lymphoma; ERK, extracellular signal-regulated kinase; SD, standard deviation; STAT, signal transducer and activator of transcription protein. ${ }^{*} \mathrm{p}<0.05$.

III/IV (HR, 12.74; 95\% CI, 1.61 to 100.74; $\mathrm{p}=0.016)$ and low serum albumin levels (HR, 3.87; 95\% CI, 1.40 to 10.72; $\mathrm{p}=0.009$ ) were significantly associated with FN based on multivariate logistic regression (Table 2, S1 Table).

\section{CIN, FN, and survival outcomes}

Survival analysis revealed that patients with CIN had a lower progression-free survival (PFS; 2-year PFS, 62.5\% vs. $74.7 \% ; \mathrm{p}=0.067$ ) and overall survival (OS; 2-year OS, 71.2\% vs. $86.4 \%$; $\mathrm{p}=0.026)$ than those without CIN. Additionally, patients with FN also had a lower PFS (2-year PFS, 51.1\% vs. $74.0 \%$; $\mathrm{p}=0.002$ ) and OS (2-year OS, $58.2 \%$ vs. $85.0 \%$; $\mathrm{p}=0.001$ ) than those without FN (Fig. 2). Analysis according to patient age showed that even if the patients' age was lower than 70 years, the PFS and OS of patients with FN were worse compared with those of patients without FN and similar to those aged 70 years or older without FN. Furthermore, all patients over 70 years of age with FN experienced relapse or died within 12 months of R-CHOP chemotherapy (Fig. 3).

\section{DLBCL cells influence the BM environment in chemo- therapy-naïve patients}

We examined the protein levels of several markers related to proliferation (pSTAT3, pAKT, pERK1/2), apoptosis (pBAD), and neutrophil release (CXCR4) in chemotherapynaïve BM cells from patients with DLBCL. Western blotting showed that the expression of pSTAT3, pAKT, pERK1/2, pBAD, and CXCR4 was increased in patients with CIN compared to that in patients without CIN (Fig. 4).

As high pSTAT3, pAKT, pERK1/2, pBAD, and CXCR4 expression in BM cells was found to be associated with the occurrence of CIN, we sought to confirm these observations in vitro by co-culturing OCI-Ly1 cells with C57BL / 6 murine BM cells using a Transwell system. We found that the expression of pSTAT3, pAKT, and pBAD proteins was upregulated in BM cells co-cultured with OCI-Ly1 cells compared to that in cells cultured alone (Fig. 5). Together with our clinical data, these results indicate that DLBCL cells may influence the BM environment, i.e., neutrophil proliferation, apoptosis, and 
$\infty$
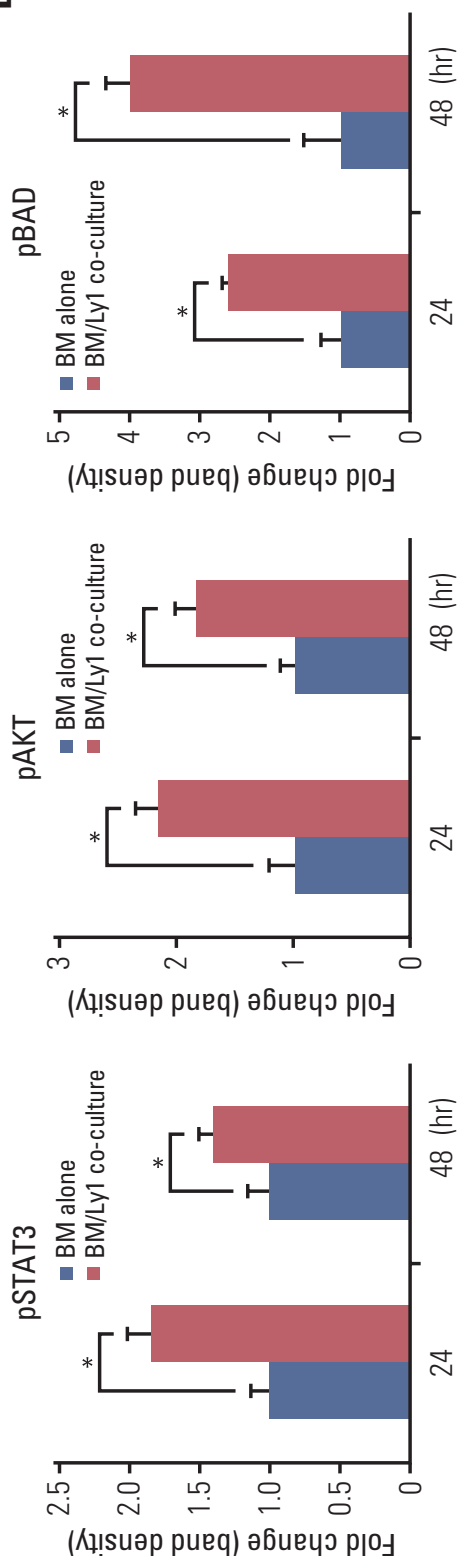

$\varangle$

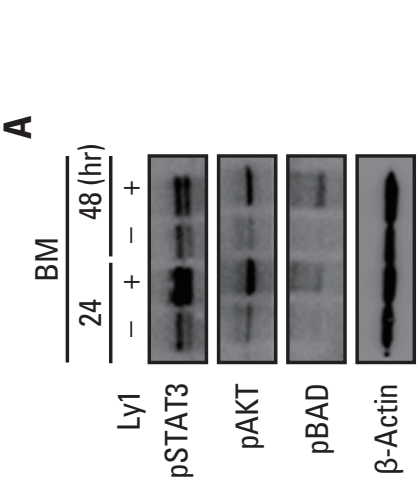

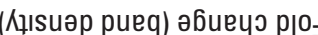

differentiation even before chemotherapy and as a result, may affect the occurrence of CIN.

\section{DLBCL cells promote neutrophil apoptosis}

To investigate whether DLBCL cells affected neutrophil apoptosis with or without chemotherapy, we examined the rate of apoptosis in neutrophils co-cultured with OCI-Ly1 cells and/or treated with doxorubicin using fluorescenceactivated cell sorting (FACS) analysis. We observed that the rate of apoptosis was higher in neutrophils co-cultured with OCI-Ly1 cells than that in neutrophils alone. The rate of apoptosis increased further after doxorubicin treatment (Fig. 6). Together, these results suggest that DLBCL cells regulate neutrophil apoptosis with or without chemotherapy, and this action can be further enhanced by chemotherapy.

\section{Discussion}

In this study, the analysis of clinical data was focused on the first cycle of chemotherapy and advanced stage DLBCL. We demonstrated that in most cases, CIN (41.8\%) and FN $(71.4 \%)$ developed during the first cycle of chemotherapy with prophylactic pegfilgrastim treatment, whereas the incidence of CIN (10\%-15.1\%) and FN (0.7\%-2.5\%) was much lower from the second cycle onwards (Fig. 1A). A Japanese study also reported similar results, wherein the incidence of FN was $9.1 \%$ (73.7\% of the cases) in the first cycle [18]. Although prophylactic pegfilgrastim appeared to reduce the risk of FN during chemotherapy, more than one-third of patients with DLBCL continued to experience CIN and around $42.3 \%$ developed FN (Fig. 1B), thus increasing the frequency of treatment-related mortality. Therefore, it is important to verify risk factors for both CIN and FN, especially during the first treatment cycle.

The independent candidate risk factors associated with FN in this study included Ann Arbor stage III/IV and hypoalbuminemia; however, previous studies have reported age $>65$ years, poor performance status, BM involvement, body mass index $<23 \mathrm{~kg} / \mathrm{m}^{2}$, renal and cardiovascular disease, relative dose intensity $>80 \%$, and no G-CSF prophylaxis as risk factors for FN [4,19-22]. Our center recently treated elderly patients with DLBCL ( $>65$ years) with a reduced dose of R-CHOP; therefore, old age was not considered as a risk factor for FN in this study. Multiple studies have shown that advanced disease status is a significant predictor of FN in various cancers, including NHL and breast, ovarian, lung, colorectal, and prostate cancers $[23,24]$. Consistent with our findings, another Korean study also demonstrated that a high Ann Arbor stage was a risk factor for CIN and FN [25]. Therefore, we investigated why advanced DLBCL (e.g., stage 

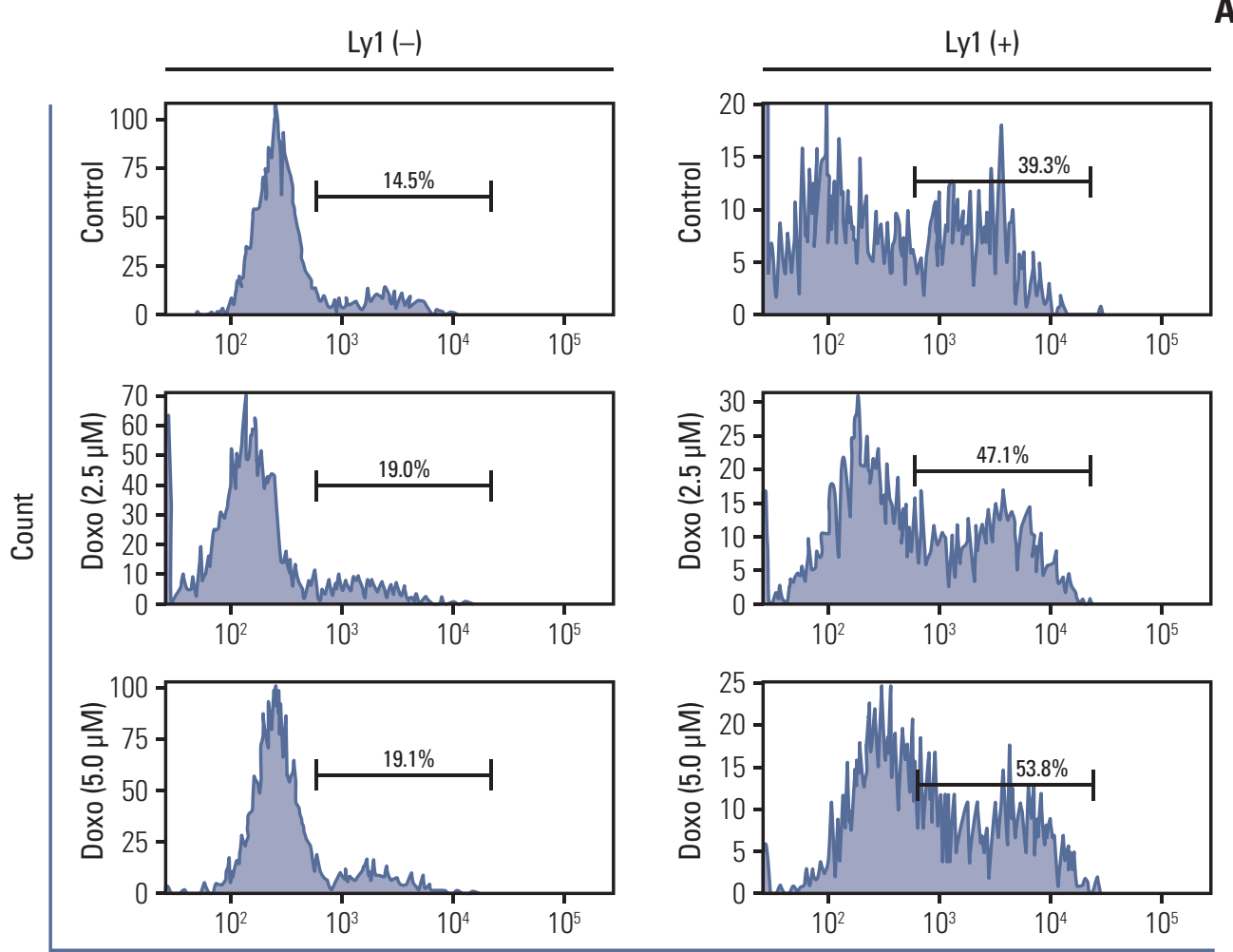

Propidium iodide

B

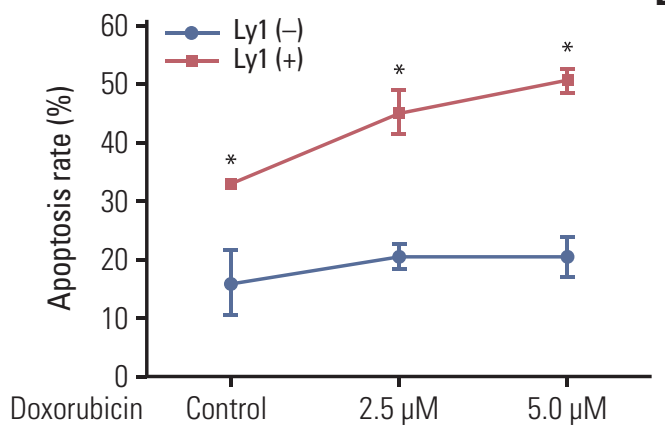

Fig. 6. DLBCL cells promote apoptosis of neutrophil during chemotherapy. Apoptotic rates of neutrophil were measured by propidium iodide followed by FACS analysis in bone marrow cells co-cultured with OCI-Ly1 for 24 hours and treated with doxorubicin $(2.5 \mu \mathrm{M}$ and $5.0 \mu \mathrm{M}$ ) for another 24 hours. (A) FACS data from a representative group is shown. (B) Quantitative data obtained from three independent FACS experiments in each group is plotted. DLBCL, diffuse large B-cell lymphoma; FACS, fluorescence-activated cell sorting. * $<0.05$.

III/IV or IPI $\geq 3$ ) is a high-risk factor for CIN and FN, even with prophylactic pegfilgrastim use during $\mathrm{R}-\mathrm{CHOP}$ chemotherapy.

Although G-CSF has no intrinsic tyrosine kinase activity, ligand binding induces a conformational change that leads to the activation of several downstream pathways, including JAK/STAT, PI3K/AKT, and mitogen-activated protein kinase (MAPK)/ERK [26-28]. Particularly, the activated GCSF receptor mediates JAK phosphorylation, which then phosphorylates the tyrosine residues of the G-CSF receptor and STAT proteins, among which STAT3 plays a pro-differentiation role in myeloid lineage development. The growthstimulatory effects of G-CSF-driven granulopoiesis depend on G-CSF/G-CSF receptor binding and the subsequent activation of STAT signaling pathways, including STAT3 activation. Indeed, in DLBCL, pSTAT3 expression is associated with an advanced stage as well as multiple extranodal sites of involvement [29], while high pAKT levels are also 
associated with a more advanced stage, two or more sites of extranodal involvement, and a higher IPI risk score [30]. Our study confirmed these results by identifying that G-CSF receptor signaling was associated with a high incidence of CIN/FN, advanced stage, and extranodal involvement (S2 Table).

Consequently, we hypothesized that advanced DLBCL influences and pre-sensitizes G-CSF receptor intracellular signaling pathways, including JAK/STAT, PI3K/AKT, and MAPK/ERK by activating cytokines such as interleukin (IL)-1, IL-6, and tumor necrosis factor- $\alpha$, which reversibly inhibit the proliferation and differentiation of myeloid progenitor cells into neutrophils via pegfilgrastim. In this study, we determined the expression of cell proliferation and survival markers such as pSTAT3, pAKT, pERK, and pBAD in $\mathrm{BM}$ cells isolated from DLBCL patients before chemotherapy. Intriguingly, these markers were overexpressed in patients with CIN compared to those without CIN after chemotherapy, thus supporting our hypothesis.

We also investigated whether DLBCL cells directly affected the BM environment to activate the intracellular G-CSF receptor signaling pathway. Our in vitro data from OCI-Ly1 cells and murine BM cells showed that pSTAT3, pAKT, and pBAD were overexpressed in BM cells co-cultured with Ly1 cells compared to BM cells cultured alone. Therefore, DLBCL cells may directly affect the proliferation and survival of $\mathrm{BM}$ progenitor cells via G-CSF receptor downstream pathways.

Consequently, we examined whether apoptosis of BM progenitor cells from patients with advanced DLBCL was affected by lymphoma cells during chemotherapy. FACS analysis demonstrated that the rate of apoptosis was higher in the neutrophil population gated on $\mathrm{Ly} 6 \mathrm{G}^{+} \mathrm{CD} 11 \mathrm{~b}^{+}$in murine $\mathrm{BM}$ cells co-cultured with OCI-Ly1 compared to those cultured alone. Furthermore, the highest rate of neutrophil apoptosis was observed when BM cells co-cultured with OCI-Ly1 were treated with doxorubicin. These results suggest that DLBCL cells induce neutrophil apoptosis and thus may affect neutrophil survival before chemotherapy.

In conclusion, we identified that advanced stage DLBCL was associated with independent predictive factors for CIN and FN even with pegfilgrastim support. It may be possible that cytokines secreted from lymphoma cells affect the BM environment and G-CSF receptor signaling pathway, which finally results in CIN and FN, even with prophylactic use of pegfilgrastim. It can be concluded that more CIN and FN events occur in the first cycle of chemotherapy, when DLBCL imposes a huge burden.

Therefore, future studies should investigate the mechanisms underlying the interaction between DLBCL cells and $\mathrm{BM}$ environment in detail to prevent CIN in patients with DLBCL. Additionally, our findings suggest that pSTAT3,
pAKT, pERK, and pBAD expression in BM cells isolated from patients with DLBCL before chemotherapy could serve as a valuable indicator for preventing neutropenia.

\section{Electronic Supplementary Material}

Supplementary materials are available at Cancer Research and Treatment website (https://www.e-crt.org).

\section{Ethical Statement}

This study was approved by the the Institutional Review Board of Pusan National University Hospital (1901-024-075). Since the data analyzed were obtained from medical records and did not include personal information, informed consent from patients was not required. All animal protocols used in this study were reviewed and approved by the Institutional Animal Care and Use Committee of Pusan National University.

\section{Author Contributions}

Conceived and designed the analysis: Kim DY, Chung JS, Kim SW, Shin HJ.

Collected the data: Kim DY, Nam J, Jeon BE, Lee JH, Jo JC.

Contributed data or analysis tools: Kim DY, Nam J.

Performed the analysis: Kim DY, Nam J.

Wrote the paper: Kim DY, Nam J, Shin HJ.

\section{ORCID iDs}

Do Young Kim ${ }^{(D)}$ : https: // orcid.org / 0000-0002-9468-8939

Jehyun Nam ${ }^{\mathbb{D}}$ : https:// orcid.org/0000-0001-8604-2313

Sang-Woo Kim (D) : https: // orcid.org/0000-0002-0507-8689

Ho-Jin Shin $\mathbb{D}$ : https:// orcid.org/0000-0002-3694-7031

\section{Conflicts of Interest}

Conflict of interest relevant to this article was not reported.

\section{Acknowledgments}

This work was supported by a 2-Year Research Grant of Pusan National University. We are grateful to the doctors who collected and analyzed the clinical data. 


\section{References}

1. Morton LM, Turner JJ, Cerhan JR, Linet MS, Treseler PA, Clarke CA, et al. Proposed classification of lymphoid neoplasms for epidemiologic research from the Pathology Working Group of the International Lymphoma Epidemiology Consortium (InterLymph). Blood. 2007;110:695-708.

2. Lee L, Crump M, Khor S, Hoch JS, Luo J, Bremner K, et al. Impact of rituximab on treatment outcomes of patients with diffuse large b-cell lymphoma: a population-based analysis. Br J Haematol. 2012;158:481-8.

3. Lindenmeyer LP, Hegele V, Caregnato JP, Wust D, Grazziotin L, Stoll P. Follow-up of patients receiving rituximab for diffuse large B cell lymphoma: an overview of systematic reviews. Ann Hematol. 2013;92:1451-9.

4. Crawford J, Dale DC, Lyman GH. Chemotherapy-induced neutropenia: risks, consequences, and new directions for its management. Cancer. 2004;100:228-37.

5. Kuderer NM, Dale DC, Crawford J, Cosler LE, Lyman GH. Mortality, morbidity, and cost associated with febrile neutropenia in adult cancer patients. Cancer. 2006;106:2258-66.

6. Bosly A, Bron D, Van Hoof A, De Bock R, Berneman Z, Ferrant $A$, et al. Achievement of optimal average relative dose intensity and correlation with survival in diffuse large B-cell lymphoma patients treated with CHOP. Ann Hematol. 2008; 87:277-83.

7. Lyman GH. Impact of chemotherapy dose intensity on cancer patient outcomes. J Natl Compr Canc Netw. 2009;7:99-108.

8. Aapro MS, Bohlius J, Cameron DA, Dal Lago L, Donnelly JP, Kearney N, et al. 2010 update of EORTC guidelines for the use of granulocyte-colony stimulating factor to reduce the incidence of chemotherapy-induced febrile neutropenia in adult patients with lymphoproliferative disorders and solid tumours. Eur J Cancer. 2011;47:8-32.

9. National Comprehensive Cancer Network. Hematopoietic growth factors (version 4.2021) [Internet]. Plymouth Meeting, PA: National Comprehensive Cancer Network; c2021 [cited 2021 May 20]. Available from: https://www.nccn.org/professionals/physician_gls/pdf/growthfactors.pdf.

10. Maher DW, Lieschke GJ, Green M, Bishop J, Stuart-Harris $\mathrm{R}$, Wolf $\mathrm{M}$, et al. Filgrastim in patients with chemotherapyinduced febrile neutropenia: a double-blind, placebo-controlled trial. Ann Intern Med. 1994;121:492-501.

11. Cooper KL, Madan J, Whyte S, Stevenson MD, Akehurst RL. Granulocyte colony-stimulating factors for febrile neutropenia prophylaxis following chemotherapy: systematic review and meta-analysis. BMC Cancer. 2011;11:404.

12. Tidow N, Welte K. Advances in understanding postreceptor signaling in response to granulocyte colony-stimulating factor. Curr Opin Hematol. 1997;4:171-5.

13. Demetri GD, Griffin JD. Granulocyte colony-stimulating factor and its receptor. Blood. 1991;78:2791-808.

14. Yang BB, Kido A. Pharmacokinetics and pharmacodynamics of pegfilgrastim. Clin Pharmacokinet. 2011;50:295-306.

15. Green MD, Koelbl H, Baselga J, Galid A, Guillem V, Gascon P, et al. A randomized double-blind multicenter phase III study of fixed-dose single-administration pegfilgrastim versus daily filgrastim in patients receiving myelosuppressive chemotherapy. Ann Oncol. 2003;14:29-35.

16. Holmes FA, O'Shaughnessy JA, Vukelja S, Jones SE, Shogan J, Savin M, et al. Blinded, randomized, multicenter study to evaluate single administration pegfilgrastim once per cycle versus daily filgrastim as an adjunct to chemotherapy in patients with high-risk stage II or stage III/IV breast cancer. J Clin Oncol. 2002;20:727-31.

17. Brusamolino E, Rusconi C, Montalbetti L, Gargantini L, Uziel L, Pinotti G, et al. Dose-dense R-CHOP-14 supported by pegfilgrastim in patients with diffuse large B-cell lymphoma: a phase II study of feasibility and toxicity. Haematologica. 2006;91:496-502.

18. Yokoyama M, Kusano Y, Takahashi A, Inoue N, Ueda K, Nishimura $\mathrm{N}$, et al. Incidence and risk factors of febrile neutropenia in patients with non-Hodgkin B-cell lymphoma receiving $\mathrm{R}-\mathrm{CHOP}$ in a single center in Japan. Support Care Cancer. 2017;25:3313-20.

19. Lyman GH, Delgado DJ. Risk and timing of hospitalization for febrile neutropenia in patients receiving CHOP, CHOP-R, or CNOP chemotherapy for intermediate-grade non-Hodgkin lymphoma. Cancer. 2003;98:2402-9.

20. Lyman GH, Morrison VA, Dale DC, Crawford J, Delgado DJ, Fridman M, et al. Risk of febrile neutropenia among patients with intermediate-grade non-Hodgkin's lymphoma receiving CHOP chemotherapy. Leuk Lymphoma. 2003;44:2069-76.

21. Morrison VA, Weller EA, Habermann TM, Li S, Fisher RI, Cheson BD, et al. Patterns of growth factor usage and febrile neutropenia among older patients with diffuse large B-cell non-Hodgkin lymphoma treated with CHOP or R-CHOP: the Intergroup experience (CALGB 9793; ECOG-SWOG 4494). Leuk Lymphoma. 2017;58:1814-22.

22. Ray-Coquard I, Borg C, Bachelot T, Sebban C, Philip I, Clapisson $\mathrm{G}$, et al. Baseline and early lymphopenia predict for the risk of febrile neutropenia after chemotherapy. Br J Cancer. 2003;88:181-6.

23. Lyman GH, Abella E, Pettengell R. Risk factors for febrile neutropenia among patients with cancer receiving chemotherapy: a systematic review. Crit Rev Oncol Hematol. 2014;90:1909.

24. Lee DG, Kim SH, Kim SY, Kim CJ, Park WB, Song YG, et al. Evidence-based guidelines for empirical therapy of neutropenic fever in Korea. Korean J Intern Med. 2011;26:220-52.

25. Choi YW, Jeong SH, Ahn MS, Lee HW, Kang SY, Choi JH, et al. Patterns of neutropenia and risk factors for febrile neutropenia of diffuse large B-cell lymphoma patients treated with rituximab-CHOP. J Korean Med Sci. 2014;29:1493-500.

26. Fukunaga R, Ishizaka-Ikeda E, Nagata S. Growth and differentiation signals mediated by different regions in the cytoplasmic domain of granulocyte colony-stimulating factor receptor. Cell. 1993;74:1079-87.

27. Santini V, Scappini B, Indik ZK, Gozzini A, Ferrini PR, Schreiber AD. The carboxy-terminal region of the granulo- 
cyte colony-stimulating factor receptor transduces a phagocytic signal. Blood. 2003;101:4615-22.

28. Palande K, Meenhuis A, Jevdjovic T, Touw IP. Scratching the surface: signaling and routing dynamics of the CSF3 receptor. Front Biosci (Landmark Ed). 2013;18:91-105.

29. Ok CY, Chen J, Xu-Monette ZY, Tzankov A, Manyam GC, Li L, et al. Clinical implications of phosphorylated STAT3 expres- sion in de novo diffuse large B-cell lymphoma. Clin Cancer Res. 2014;20:5113-23.

30. Hong JY, Hong ME, Choi MK, Kim YS, Chang W, Maeng CH, et al. The impact of activated p-AKT expression on clinical outcomes in diffuse large B-cell lymphoma: a clinicopathological study of 262 cases. Ann Oncol. 2014;25:182-8. 